\title{
Tecnologias digitais na gestão escolar: Práticas, proficiência e necessidades de formação dos diretores escolares em Portugal
}

\author{
João Piedadei \& Neuza Pedro ${ }^{i}$ \\ Universidade de Lisboa, Portugal
}

\begin{abstract}
Resumo
O presente artigo apresenta os resultados de uma investigação de cariz descritivo, desenvolvida junto de 133 diretores de escolas e agrupamentos de escolas do ensino público de Portugal continental, onde se procurou analisar o índice de utilização das tecnologias digitais (TD) nas práticas de gestão escolar e o sentido de autoeficácia na utilização das mesmas, apresentados pelos diretores escolares. O processo de recolha de dados estruturou-se com base na aplicação de duas escalas organizadas num único instrumento disponibilizado online: a 'Computer self-efficacy scale' e a ‘Escala de utilização das TD nas práticas de gestão escolar', no ano letivo 2012-2013. Os resultados tendem a evidenciar que os diretores escolares apresentam scores médios favoráveis de autoeficácia e de utilização das TD nas suas práticas profissionais. O estudo procurou ainda explorar as necessidades de formação na área das TD e, apesar dos scores favoráveis de proficiência e utilização das mesmas nas práticas profissionais, os diretores referem importantes necessidades de formação.
\end{abstract}

Palavras-chave

Tecnologias digitais na educação; Diretores escolares; Autoeficácia; Utilização das TD na gestão escolar

\section{Introdução}

O presente estudo enquadra-se no domínio das tecnologias digitais (TD) na educação, com foco principal na utilização das mesmas nas práticas 
profissionais dos diretores escolares. Assumiu-se pertinente estudar as crenças e perceções das práticas dos diretores escolares na utilização das TD pelo papel que os mesmos exercem enquanto promotores do processo de integração das tecnologias nas práticas profissionais dos professores das suas escolas, potenciando, deste modo, a modernização de práticas de ensino e de aprendizagem, da gestão da informação e comunicação entre os vários agentes educativos em contexto escolar.

Este estudo empírico, de natureza quantitativa, procura contribuir para a análise dos níveis de proficiência e utilização das TD nas práticas profissionais dos diretores de escolas ou agrupamentos de escolas do ensino público português e, ao mesmo tempo, contribuir para a identificação de um conjunto de fatores condicionantes de uma mais eficiente integração das mesmas nos contextos escolares, bem como para a sinalização das necessidades de formação, na área da temática em estudo, sentidas pelos diretores escolares.

As potencialidades educativas das tecnologias estão hoje em dia estudadas e publicadas em literatura de referência; no entanto, o seu processo de integração não é simples, existindo um conjunto de fatores inibidores ou obstáculos que é necessário considerar. Esses obstáculos à integração das TD têm sido o foco de vários estudos nos últimos anos. Em diversos trabalhos científicos foram já apresentados obstáculos de diversa ordem, sendo em todos eles a falta de formação de professores apontada como um dos principais fatores inibidores, tanto em contexto nacional como internacional (Bravo \& Fernández, 2009; Costa, 2008; Culp, Honey, \& Mandinach, 2003; Miranda, 2007; Norris, Soloway, \& Sullivan, 2002). Assim, vários autores confirmam que, com vista a implementar uma real e efetiva integração educativa das tecnologias, o desenvolvimento de competências tecnológicas por parte dos profissionais da educação deverá ser um dos principais aspetos a garantir (Gooler, Kautzer, \& Knuth, 2000; Ivers, 2002; Liu \& Huang, 2005).

Pedro (2011) procurou organizar os obstáculos à integração das TD em contexto escolar organizando-os em 4 níveis de análise: (i) nível macrossistémico: com uma dimensão societal e onde se integram elementos relacionados com as ideologias sociopolíticas, sistema de crenças e formas de organização social estabelecidas; inserem-se neste domínio fatores de 
âmbito conjuntural, diretamente relacionados com o sistema e as políticas educativas; (ii) nível exossistémico: relacionado com fatores de âmbito institucional, isto é, ligados às dinâmicas organizacionais das instituições escolares, considerando as definições e medidas assumidas nas escolas; (iii) nível mesossistémico: associado a fatores comuns à classe docente, ao corpus profissional de que cada professor faz parte e que determina elementos comuns a todos os professores, nomeadamente definição dos estatutos da carreira docente, programas de formação para professores, etc., e que exercem influência ao nível do profissionalismo e identidade docente; e (iv) nível microssistémico: organiza-se em torno de elementos associados à dimensão inter-relacional do sujeito, ou seja, o seu sistema de crenças, as suas atitudes, aspirações, motivações, receios, práticas e competências.

O processo de integração efetiva das tecnologias nas escolas requer a conjugação favorável de todas as variáveis e fatores apresentados, envolvendo escolas, professores, líderes escolares, alunos, encarregados de educação e decisores políticos. O presente trabalho assume como foco de estudo os líderes escolares, entendendo-os como atores frequentemente negligenciados e pouco envolvidos neste processo.

\section{Diretores escolares: Responsabilidades na integração das tecnologias em contexto escolar}

A atual definição de diretor escolar foi criada pelo Decreto-Lei $n^{\circ}$ $75 / 2008$, de 22 de abril, substituindo a designação de presidente do conselho executivo. Deste modo, o órgão de gestão deixa de ser um órgão colegial para se tornar num órgão de administração unipessoal, onde as competências e autonomia do diretor são reforçadas. As competências e perfil profissionais dos diretores escolares estão definidas nos Decretos-Lei $n^{\circ} 75 / 2008$, de 22 de abril, e $n^{\circ} 137 / 2012$, de 2 de julho. Nestes normativos assume-se como objetivo reforçar a autonomia e a capacidade de intervenção dos órgãos de direção das escolas para reforçar a eficácia da execução das medidas de política educativa e da prestação do serviço público de educação. Ao diretor escolar é atribuída a gestão financeira, administrativa e pedagógica da escola ou agrupamento de escolas. Enquanto entidade máxima na gestão escolar, assume um papel de relevo em todas as áreas da administração escolar, incluindo as relacionadas com a integração educativa das tecnologias. 
No âmbito do Plano Tecnológico da Educação (PTE) foram criadas pelo Despacho $n^{\circ} 700 / 2009$, de 9 de janeiro, as equipas PTE, com o objetivo de coordenar e acompanhar a implementação e desenvolvimento dos projetos relacionados com O PTE. O diretor escolar assume por inerência a coordenação destas equipas e a responsabilidade de nomear os membros das mesmas de entre docentes com competências técnicas e pedagógicas para desenvolver e supervisionar projetos no âmbito da integração das TIC; responsável pelos serviços administrativos; não docentes com competências TIC; alunos estagiários de cursos profissionais; coordenador da biblioteca escolar; e outros elementos que considere competentes no uso das tecnologias. O diretor escolar deve ainda nomear um responsável técnico e um responsável pedagógico para acompanhamento das iniciativas e projetos PTE ou TIC na escola.

Tendo em consideração as competências atribuídas ao diretor escolar, reforçadas nos Decretos-Lei n 75/2008, de 22 de abril, e no 137/2012, de 2 de julho, e as definidas no âmbito do Plano Tecnológico da Educação, o diretor escolar assume responsabilidade sobre todas as questões relacionadas com a definição das estratégias e planos de intervenção ao nível da gestão pedagógica e tem um papel preponderante na integração educativa das TD no seu contexto escolar.

Vários têm sido os estudos que relacionam a problemática da gestão e da liderança escolar e a efetiva integração das tecnologias, quer ao nível curricular e pedagógico quer ao nível da gestão e da comunicação institucional (Costa, 2008; Drent \& Meelissen, 2008; Stuart, Mills, \& Remus, 2009; Tondeur, Keer, Braak, \& Valcke, 2008). Na verdade, segundo Jacobson e Hunter (2004), os líderes escolares têm vindo a sofrer uma crescente pressão no que se relaciona com a integração das tecnologias por parte da comunidade educativa. Consideram os mesmos autores que, embora a mudança implique um esforço conjunto de toda a comunidade educativa, o papel dos líderes escolares é imprescindível. Os líderes escolares serão os responsáveis pela identificação e definição de estratégias de mudança que serão necessárias nos seus contextos educativos (Stuart et al., 2009) para que qualquer mudança de práticas se instale.

Embora a organização da escola seja, no todo, importante, a liderança é, no seu interior, um elemento crítico no que se refere à integração da 
tecnologia na cultura da escola (Anderson \& Dexter, 2000). Segundo Seweeney (2005), "uma liderança eficaz é a componente mais crítica para garantir o sucesso na implementação de qualquer programa num ambiente educacional" (p. 48). As ações e visões estratégicas dos líderes escolares têm grande impacto e podem influenciar a inovação educativa não apenas no panorama institucional mas igualmente sob uma perspetiva societal.

De forma indireta, vários outros autores referem também o papel dos líderes escolares na liderança e na motivação de todos os agentes educativos da sua comunidade escolar para encetar as mudanças exigidas pela sociedade tecnológica e todas as suas implicações para a escola (Fullan, 2001; Hayes, 2007; OCDE, 2001).

Num estudo realizado em 2000 em escolas irlandesas, Mulkeen (2000) constatou que as práticas de utilização das TD por parte dos líderes escolares exercem marcada influência nas práticas de utilização das mesmas por parte dos restantes professores. O mesmo autor salientou ainda como fator favorável a participação das escolas em projetos-piloto inovadores, enunciando que o grau de envolvimento das escolas em tais projetos se apresenta ele mesmo intrinsecamente ligado ao estímulo promovido e às favoráveis condições criadas pelas lideranças escolares.

Já em 2010, e com base nos resultados do programa Preparing Tomorrows Teachers to Teach with Technology (PT3), Polly, Mims, Sheperd, e Inan (2010) sinalizam igualmente efeitos muito positivos nas práticas docentes de projetos no âmbito das TD que foram considerados prioritários pelos respetivos administradores ou diretores escolares.

Também Tearle (2003) alertou, nos seus estudos, para o facto de o contexto-escola como um todo desempenhar uma forte influência na utilização das TIC na sala de aula e, em particular, salientou como exercendo um papel importante no incentivo à utilização das tecnologias (i) o apoio por parte dos órgãos de direção escolar e (ii) as perspetivas construtivistas acerca da aprendizagem. Em sentido semelhante, Younie (2006) e Wong e Li (2008) sublinham igualmente a importância assumida pelas direções escolares na integração educativa das tecnologias, defendendo a necessidade de ver estimulado e instituído, nas escolas, lideranças transformacionais, ou seja, um estilo de liderança que coloca o foco nos compromissos e capacidades da organização para inovar e reestruturar a sua prática. Como igualmente 
determinante do processo de inovação tecnológica, Wong e Li (2008) destacam ainda a importância que assume o clima escolar, o ambiente social vivido e o grau em que o mesmo se revela favorável/desfavorável à integração tecnológica. Também Afshari, Abu Bakar, Luan, Abu Samah, e Fooi (2008) apontam como fator crítico para a transformação tecnológica do currículo escolar o papel do diretor escolar, reforçando a necessidade de desenvolver programas de formação e liderança transformacional, gestão e tecnologia.

O papel exercido pelos órgãos de gestão escolares foi igualmente assinalado por Daly, Pachler, e Pelletier (2009). Estes autores referem especificamente como grande desafio o desenvolvimento, junto das lideranças escolares, de uma 'visão' apropriada acerca do papel das TIC no contexto escolar concreto. Complementarmente, Cakir (2012) enuncia que no seio dos papéis que se requer que os diretores escolares assumam surge a 'liderança tecnológica' (technology leadership), sendo responsáveis pela prioridade dada à integração das TD nas suas escolas. De acordo com o autor, "being a technology leader should entail responsibilities such as acting as a role model, providing encouragement and direction, sharing knowledge and information and (...) they are also expected to be highly proficient in using technology" (Cakir, 2012, p. 279).

\section{Formação e programas de promoção de competências (inovadoras) na gestão}

Apesar da importância reconhecida pela literatura de referência aos diretores escolares no processo de integração das tecnologias nas escolas, os vários programas de estímulo à modernização em contexto escolar definidos a nível nacional têm vindo a não contemplar ações concretas dirigidas a diretores escolares. Salienta-se, em particular, a limitada oferta de ações ao nível da formação em gestão e liderança com tecnologia ou programas de apoio. Os diretores escolares, enquanto professores, podem, nos últimos anos, ter frequentado iniciativas de formação no âmbito das TD definidas para os seus grupos disciplinares e não direcionadas diretamente para a gestão; contudo, entende-se que formação específica seria o desejável. 
Retrocedendo no tempo, sinaliza-se que no ano de 2007 a formação contínua de professores na área das tecnologias foi considerada prioritária, tendo sido criadas 5 áreas de intervenção distintas: (i) Animação e dinamização de projetos TIC nas escolas; (ii) A utilização das TIC nos processos de ensino e aprendizagem; (iii) Fatores de liderança na integração das TIC na escola; (iv) Utilização das TIC em contextos inter e transdisciplinares; e (v) Os novos programas na área da informática. Todas estas áreas deram origem a ações de formação creditadas junto do Conselho Científico-Pedagógico da Formação Contínua (CCPFC). Destaca-se a terceira área de intervenção, relativa a fatores de liderança na integração das TIC na escola, que assumia como destinatários privilegiados diretores e membros da direção das escolas e/ou agrupamentos de escolas. Entre outros objetivos, pretendia-se motivar desta forma os órgãos de gestão para a importância das TIC e promover o desenvolvimento de uma visão e objetivos de liderança tecnológica para as suas instituições ${ }^{1}$.

De 2007 até à atualidade, não se registam outros movimentos de apoio e estímulo ao envolvimento e responsabilização dos diretores pelo processo de integração das TD nas escolas. Aliás, através de pesquisa de ações de formação creditadas pelo Conselho Científico-Pedagógico da Formação Contínua (CCPFC), disponíveis no espaço online deste organismo tutelado pelo Ministério da Educação e da Ciência, verifica-se que nos últimos 10 anos não têm sido creditadas ações de formação na área das TD especificamente dirigidas a diretores escolares. Para este público em particular, identificou-se apenas a creditação de ações de formação relacionadas com autoavaliação das escolas, gestão administrativa e contratação pública, sendo que se revelam praticamente inexistentes iniciativas relacionadas com a integração das TD em particular ou com áreas análogas, como sejam: e-gestão, eliderança, inovação na gestão escolar, e-comunicação.

Como exceção ao acima exposto sinaliza-se o projeto "Líderes Inovadores", desenvolvido pela parceria entre a Microsoft Portugal e o Ministério da Educação, com o objetivo de promover o desenvolvimento de competências de liderança e gestão inovadoras junto dos líderes escolares (DGRHE, 2012). A iniciativa alicerça-se sobretudo na planificação e desenvolvimento de um programa de formação com um total de 150 horas, onde colaboram vários especialistas reconhecidos no plano nacional e 
internacional, e estruturado em torno de diversas áreas do saber: (i) Utilização das tecnologias nas escolas; (ii) A mudança e as competências do século XXI; (iii) Avaliação; (iv) Definição de objetivos e planeamento estratégico; (v) Gestão, inovação, criatividade e mudança organizacional; (vi) Gestão de recursos humanos; (vii) Liderança e construção de equipas; e (viii) Comunicação. O programa encontra-se atualmente na $4^{\text {a }}$ edição, para a qual foram selecionados 100 diretores de escolas e/ou agrupamentos de escolas (DGAE, 2013b). Nas três edições anteriores do programa participaram cerca de 250 diretores de escolas e/ou agrupamentos de escolas, o que deu origem a 203 estudos de caso disponíveis online ${ }^{2}$ que apresentam a planificação e implementação do plano de mudança e inovação que cada diretor escolar desenvolveu e aplicou na sua instituição.

\section{Metodologia}

\subsection{Objetivos do estudo}

Contextualizado no domínio da integração das TD nas escolas portuguesas e focalizado, em particular, nas práticas dos diretores escolares, o presente estudo pretende analisar o papel exercido pelos diretores escolares no processo de integração das tecnologias nas práticas escolares. O propósito central da investigação procura ser atingido através de: (i) análise do grau de proficiência na utilização das TD apresentado pelos diretores escolares, operacionalizado através do estudo do sentido de autoeficácia no uso das tecnologias evidenciado pelos mesmos; (ii) análise do grau de utilização das TD nas práticas de gestão escolar referido pelos diretores escolares; (iii) análise da relação entre os constructos anteriores; e (iv) identificação de fatores potenciadores e limitadores da integração educativa das TD na perspetiva dos diretores escolares, com igual sinalização de necessidades de formação na área das TD evidenciadas pelos mesmos.

\subsection{Design metodológico}

Considerando a problemática de investigação e os objetivos definidos desenvolveu-se uma investigação empírica de natureza quantitativa. A investigação em causa enquadra-se assim no paradigma de investigação póspositivista (Creswell, 2010), assumindo-se como uma investigação de 
natureza descritiva, onde se procurou garantir um modo de atuação objetivo e não intervencionista por parte dos investigadores (Creswell, 2003).

Deste modo, a pesquisa em causa baseou-se na obtenção de indicadores numéricos representativos das variáveis em estudo, em particular:

(i) sentido de autoeficácia na utilização das $T D$, operacionalizado através do score total médio obtido na escala psicométrica selecionada para o efeito: a 'Computer self-efficacy scale' (Cassidy \& Eachus, 2002). O conceito de autoeficácia surge amplamente descrito e estudado na teoria cognitiva social de Bandura (1977), tendo sido apresentado pelo autor através da ideia de "self-directed mastery", i.e., a capacidade do ser humano em auto-orientar e dirigir ativamente o seu comportamento para a mestria, a procura de excelência na sua atuação. Vários estudos têm, nos últimos anos, apontado o sentido de autoeficácia ou os aspetos motivacionais como um dos fatores condicionantes à integração educativa das TD, com foco na sua utilização em sala de aula (Mueller, Wood, Willoughby, Ross, \& Specht, 2008; Paraskeva, Bouta, \& Papagianni, 2008; Vannatta \& Fordham, 2004; Woodbridge, 2004). Investigações recentes, desenvolvidas em contexto nacional, apontam de igual modo a autoeficácia dos professores como um fator preponderante na integração das TD em contexto escolar, na medida em que níveis mais elevados de autoeficácia encontram-se diretamente ligados a níveis mais elevados de atuação com as mesmas (Piedade, 2010; Piedade \& Pedro, 2012; Pedro, 2011; Santos, 2010);

(ii) utilização das TD nas práticas de gestão escolar, operacionalizada através do score total médio obtido na escala de utilização das TD nas práticas de gestão escolar - escala desenvolvida pelos investigadores no âmbito da investigação em causa;

(iii) necessidades de formação na área das TD evidenciadas pelos diretores escolares;

(iv) fatores potenciadores e limitadores da integração educativa das TD sinalizados pelos diretores escolares. 


\subsection{Instrumentos de recolha de dados}

O processo de recolha de dados estruturou-se com base na aplicação das duas escalas já referidas organizadas num único instrumento disponibilizado online.

A 'Computer Self-Efficacy Scale' foi desenvolvida por Cassidy e Eachus (2002), cumprindo os pressupostos de aplicação da teoria sociocognitiva de Bandura, sendo apresentada pelos autores como "domainspecific". A escala, na sua versão original, encontra-se estruturada em 30 itens, com 5 opções de resposta de formato Likert (variando entre "Discordo totalmente" e "Concordo totalmente"), apresentando 15 itens formulados de forma positiva e 15 itens formulados de forma negativa. A análise da qualidade métrica da 'Computer Self-Efficacy Scale' foi previamente realizada em estudos anteriores, tendo apresentado um coeficiente alfa de Cronbach de .94, revelando um elevado índice de fiabilidade (Piedade, 2010; Pedro, 2011).

A 'Escala de utilização das TD nas práticas de gestão escolar' apresenta-se como um instrumento multidimensional, onde se considera que a utilização das tecnologias pelos diretores escolares ocorre com frequência e intensidade diferenciada nas várias dimensões das suas práticas profissionais. Deste modo, a escala estrutura-se com base nas dimensões apresentadas no quadro 1, o que permite o cálculo do score global da escala e o score de cada uma das dimensões.

Os itens da escala assumem um formato de resposta de escolha múltipla, sendo solicitado aos diretores que selecionem uma opção de resposta numa escala que varia entre "Muito raramente" a "Muito frequentemente". As respostas são cotadas com valores situados entre 1 e 5 , respetivamente.

O questionário integra ainda um conjunto de itens relativos a constrangimentos verificados na integração das tecnologias na escola e a necessidades de formação identificadas no âmbito da utilização das tecnologias nas suas práticas profissionais. 


\section{Quadro 1 - Dimensões da escala de utilização das tecnologias digitais} nas práticas de gestão escolar

\begin{tabular}{|c|c|c|}
\hline & Dimensão & Item Exemplo \\
\hline 1 & $\begin{array}{l}\text { Comunicação: Utilização das TIC pelos } \\
\text { diretores para comunicação com vários } \\
\text { agentes educativos e com a } \\
\text { comunidade }\end{array}$ & $\begin{array}{l}\text { Q22 - Com que frequência utiliza as TIC para comunicar } \\
\text { institucionalmente com organismos locais (ex. Municípios, } \\
\text { Juntas de Freguesia, Empresas e outros parceiros)? }\end{array}$ \\
\hline 2 & $\begin{array}{l}\text { Planificação: Utilização das TIC para } \\
\text { planificaçăo da atividade profissional }\end{array}$ & $\begin{array}{l}\text { Q12 - Com que frequência utiliza as TIC para gestão da } \\
\text { agenda de trabalho? }\end{array}$ \\
\hline 3 & $\begin{array}{l}\text { Dinamização de reuniões: Utilização } \\
\text { das TIC para dinamização e condução } \\
\text { de reuniões de trabalho }\end{array}$ & $\begin{array}{l}\text { Q13 - Com que frequência utiliza as TIC para realização de } \\
\text { apresentações eletrónicas nas reuniões dos órgãos da } \\
\text { escola/agrupamento? }\end{array}$ \\
\hline 4 & $\begin{array}{l}\text { Avaliação: Utilização das TIC para } \\
\text { avaliação de pessoal docente e não } \\
\text { docente e autoavaliação de escola }\end{array}$ & $\begin{array}{l}\text { Q14 - Com que frequência utiliza as TIC para a análise } \\
\text { estatística dos resultados escolares? }\end{array}$ \\
\hline 5 & $\begin{array}{l}\text { Gestão administrativa: Utilização TIC } \\
\text { para realização de atividades de } \\
\text { gestão }\end{array}$ & $\begin{array}{l}\text { Q16 - Com que frequência utiliza plataformas e aplicações } \\
\text { online para envio de dados oficiais solicitados pela Tutela (ex. } \\
\text { estatísticas de } n^{\circ} \text { alunos, professores, etc...?) }\end{array}$ \\
\hline
\end{tabular}

$\mathrm{Na}$ fase de construção da 'Escala de utilização das TD pelos diretores escolares', procedeu-se à pré-testagem da mesma. Este processo foi organizado em duas fases: validação por especialistas e aplicação-piloto do instrumento. No processo de validação por especialistas, solicitou-se a análise da escala e respetivas dimensões a três académicos da área da gestão e administração escolar de três instituições de ensino superior em Portugal (um da Universidade de Lisboa, um do Instituto Piaget e um do Instituto Politécnico de Santarém). Após a análise dos contributos dos especialistas, procedeu-se à reformulação de dois itens da escala e à eliminação de um outro, dando origem à segunda versão do instrumento. A segunda versão da escala foi validada junto de 10 diretores de escolas ou agrupamentos de escolas com pelo menos 8 anos de experiência no cargo. A análise da qualidade métrica da escala revelou um coeficiente alpha de Cronbach representativo de uma elevada fiabilidade do instrumento em causa $(r=.97)$, de acordo com Hill e Hill (2008). 


\subsection{Participantes e procedimentos}

Os diretores de escolas e agrupamentos de escolas do ensino público de Portugal Continental surgem como participantes do presente estudo. Considerando a natureza do mesmo, e por se pretender obter respostas do maior número de diretores possível, optou-se por inquirir a totalidade dos diretores escolares. O estudo em causa foi realizado em 2012/13 e, de acordo com os dados então disponíveis, existiam 831 agrupamentos de escolas ou escolas não agrupadas em Portugal Continental (DGAE, 2013a). O processo de recolha de dados realizou-se entre fevereiro e abril de 2013 e obteve-se respostas de 133 diretores escolares, correspondendo a $16 \%$ do grupo populacional em estudo.

O grupo de participantes apresenta uma distribuição equilibrada quanto ao género, verificando-se uma ligeira predominância de elementos do género masculino, correspondendo a $52.63 \%$ dos inquiridos. A maioria dos sujeitos apresenta idades compreendidas entre os 51 e os 60 anos (51.13\%); $78.95 \%$ lecionam há mais de 20 anos e $67.67 \%$ pertencem à direção da escola há mais de 11 anos. Dos diretores inquiridos, $65.41 \%$ apresentam como habilitações académicas o grau de licenciatura e $75.19 \%$ possuem formação especializada em administração e gestão escolar. No grupo de diretores respondentes estão representadas todas as áreas científicas e disciplinares, verificando-se uma clara predominância para as ciências sociais e humanas $(36.84 \%)$ e ciências exatas e experimentais $(20.30 \%)$. A distribuição geográfica dos participantes, de acordo com Direção de Serviços Regionais (DSR) e Quadros de Zona Pedagógica (QZP), encontra-se representada no quadro 2. Participaram neste estudo $41.03 \%$ do total de diretores da DSR Algarve, $25.35 \%$ da DSR Alentejo, $18.18 \%$ da DSR Lisboa e Vale do Tejo, $14.61 \%$ da DSR Centro e $8.58 \%$ da DSR do Norte. 
Quadro 2 - Distribuição geográfica dos participantes

\begin{tabular}{|c|c|c|c|c|c|}
\hline \multicolumn{2}{|c|}{ Direção de Serviços Regionais } & \multirow{2}{*}{$\begin{array}{c}\text { QZP } \\
\text { QZP1 }\end{array}$} & \multirow{2}{*}{$\begin{array}{c}\text { Total } \\
219\end{array}$} & \multirow{2}{*}{$\begin{array}{c}\mathbf{n} \\
18\end{array}$} & \multirow{2}{*}{$\begin{array}{c}\% \\
8.22 \% \\
\end{array}$} \\
\hline $858 \%$ & RSR Norto & & & & \\
\hline $8.58 \%$ & DSR Norte & QZP2 & 49 & 5 & $10.20 \%$ \\
\hline & & QZP3 & 78 & 9 & $11.54 \%$ \\
\hline \multirow[t]{2}{*}{$14.61 \%$} & DSR Centro & QZP4 & 59 & 7 & $11.86 \%$ \\
\hline & & QZP5 & 41 & 10 & $24.39 \%$ \\
\hline \multirow{2}{*}{$18.18 \%$} & \multirow{2}{*}{ DSR Lisboa e Vale do Tejo } & QZP6 & 73 & 17 & $23.29 \%$ \\
\hline & & QZP7 & 202 & 33 & $16.34 \%$ \\
\hline \multirow{2}{*}{$25.35 \%$} & \multirow{2}{*}{ DSR Alentejo } & QZP8 & 40 & 11 & $27.50 \%$ \\
\hline & & QZP9 & 31 & 7 & $22.58 \%$ \\
\hline $41.03 \%$ & DSR Algarve & QZP10 & 39 & 16 & $41.03 \%$ \\
\hline \multicolumn{3}{|c|}{ TOTAL } & 831 & 133 & $16.00 \%$ \\
\hline
\end{tabular}

Previamente ao início do processo de recolha de dados realizaram-se todas as diligências formais para a recolha de dados em ambiente escolar nacional. Nesse sentido, procedeu-se à submissão dos instrumentos anteriormente referidos à Direção-Geral de Educação com o pedido de autorização para recolher dados junto dos diretores das escolas públicas nacionais (deferido com o $\mathrm{N}^{\circ} 0346000001$ ).

Os instrumentos foram organizados numa plataforma online opensource, que permite a exportação de dados para as várias aplicações de análise estatística, e o convite de participação no estudo foi enviado aos participantes através do correio eletrónico institucional das escolas.

No processo de organização dos dados obtidos procedeu-se, numa primeira fase, à exportação dos mesmos para uma folha de cálculo. Após a organização dos dados e a eliminação de submissões nulas, duplas submissões e dados irrelevantes para a investigação em causa, os dados foram importados para o software IBM SPSS Statistics, versão 21.

\section{Resultados}

Tendo em consideração as variáveis em análise - sentido de autoeficácia na utilização das tecnologias e índice de utilização das mesmas nas práticas profissionais dos diretores escolares -, os dados que a seguir se apresentam organizam-se segundo: (i) nível de proficiência e índice de utilização das tecnologias nas várias dimensões da atividade profissional dos 
diretores escolares e respetivos coeficientes de correlação; (ii) relação entre os constructos em análise; (iii) fatores potenciadores da integração educativa das tecnologias segundo a perspetiva dos diretores escolares e necessidades de formação na área das TD sinalizadas pelos mesmos.

\subsection{Autoeficácia e utilização das tecnologias digitais pelos diretores escolares}

Pela análise dos score médios da tabela 3 , constata-se, relativamente ao nível de proficiência, que os diretores escolares apresentam um valor médio de 3.95, o que revela um sentido de autoeficácia na utilização das TD favorável, tendo em consideração que os valores da escola estavam compreendidos entre 1, indicando uma reduzida perceção de autoeficácia, e 5, significando uma elevada perceção de autoeficácia. Em sentido semelhante, para a variável 'utilização das tecnologias nas práticas profissionais', o score médio da escala (4.22) apresenta-se revelador de um nível elevado da utilização das TD.

Analisando os resultados encontrados para as várias dimensões da escala de utilização das TD, verificam-se diferenças nos valores médios apresentados em cada dimensão, embora os dados permitam identificar níveis favoráveis de utilização em todas as dimensões sob análise. Analisando discriminadamente os valores médios encontrados para cada uma das dimensões, verifica-se que a dimensão 'gestão administrativa' apresenta um score mais elevado (4.45), revelando uma forte utilização das tecnologias no apoio à gestão escolar. Em sentido oposto, a dimensão de 'comunicação' apresenta o score mais baixo (3.98), indicando, ainda assim, um nível de utilização favorável. Os valores encontrados para o desvio-padrão apresentam-se próximos de zero, revelando pouca variância nas respostas dos sujeitos. 
Quadro 3 - Valores médios e desvio-padrão das escalas de proficiência e de utilização das tecnologias digitais

\begin{tabular}{lcc}
\hline \multicolumn{1}{c}{ VARIÁveIS } & MÉDIA & DESVIO-PADRÃo \\
\hline Nível de Proficiência & 3.95 & .48 \\
Utilização das TD & 4.22 & .64 \\
\hline \multicolumn{1}{c}{ DIMENSõES } & \\
\hline Dimensão 1 - Comunicação & 3.98 & .66 \\
Dimensão 2 - Planificação & 4.25 & .72 \\
Dimensão 3 - Reuniões & 4.13 & .81 \\
Dimensão 4 - Avaliação & 4.23 & .80 \\
Dimensão 5 - Gestão administrativa & 4.45 & .81 \\
\hline
\end{tabular}

Com o propósito de analisar a relação entre o grau de proficiência e o índice de utilização das TD, nas várias dimensões, apresentados pelos diretores escolares, procedeu-se à análise dos coeficientes de correlação de Pearson, encontrados entre os scores médios dos constructos em análise.

Quadro 4 - Coeficientes de correlação entre o nível de proficiência, índice de utilização das tecnologias digitais e respetivas dimensões

\begin{tabular}{|c|c|c|c|c|c|c|}
\hline & $\begin{array}{l}\text { UTILIZAÇÃO } \\
\text { DAS TD }\end{array}$ & $\begin{array}{c}\text { DIMENSÃo1 } \\
\text { CoMUNICAÇÃo }\end{array}$ & $\begin{array}{l}\text { DIMENSÃO2 } \\
\text { PLANIFICAÇÃo }\end{array}$ & $\begin{array}{c}\text { DIMENSÃo3 } \\
\text { REUNIÕES }\end{array}$ & $\begin{array}{l}\text { DIMENSÃo4 } \\
\text { AvaLIAÇÃo }\end{array}$ & $\begin{array}{c}\text { DIMENSÃ 05 GESTÃo } \\
\text { ADMINISTRATIVA }\end{array}$ \\
\hline $\begin{array}{c}\text { Nível de } \\
\text { Proficiência }\end{array}$ & $.33^{* *}$ & $.27^{* *}$ & $.39^{* *+}$ & $.30^{* *+}$ & $.31^{* *}$ & $.19^{*}$ \\
\hline Utilização das TD & & .87 & .77 & .82 & .90 & 85 \\
\hline $\begin{array}{c}\text { Dimensão1 } \\
\text { Comunicação }\end{array}$ & & & $.61^{\prime \prime}$ & $.66^{* *}$ & $.72^{* *}$ & .66 \\
\hline $\begin{array}{l}\text { Dimensão2 } \\
\text { Planificação }\end{array}$ & & & & $.74^{2 \pi}$ & $.59^{\pi x}$ & $.48^{\pi x}$ \\
\hline $\begin{array}{c}\text { Dimensão3 } \\
\text { Reuniões }\end{array}$ & & & & & $.61^{* *}$ & $.52^{* *+}$ \\
\hline $\begin{array}{c}\text { Dimensão4 } \\
\text { Avaliação }\end{array}$ & & & & & & $.83^{* *}$ \\
\hline
\end{tabular}

Da observação dos coeficientes de correlação apresentados no quadro 4, verifica-se que as variáveis se correlacionam de forma positiva e significativa. As variáveis 'níveis de proficiência' e 'índice de utilização das TD' apresentam um coeficiente de correlação significativo $(r=.33)$, sendo indicador de correlação entre as variáveis. Em sentido semelhante, é igualmente 
possível analisar as correlações encontradas entre os valores médios do índice de utilização das TD e todas as dimensões da escala.

\subsection{Fatores potenciadores da integração educativa das tecnologias digitais e necessidades de formação}

Com o objetivo de identificar fatores preponderantes e potenciadores de uma efetiva integração das TD nas escolas e agrupamentos de escolas, solicitou-se aos diretores que selecionassem os fatores que consideram ter maior impacto na integração das tecnologias nas práticas escolares. A análise do quadro 5 mostra que os diretores inquiridos consideram que os fatores que poderão ter um impacto mais significativo na integração das TD nas escolas são a existência de professores inovadores (68.42\%), a valorização do trabalho dos professores $(54.89 \%)$ e a disponibilização de mais software específico para as várias disciplinas (52.63\%). Em sentido oposto, referem a realização de mais ações de formação de âmbito generalista (15.04\%), a flexibilização dos currículos $(24,81 \%)$ e as políticas educativas e incentivos estruturais (30.83\%) como os fatores que consideram ter menor relevância na adoção e integração das TD nas escolas.

Uma das premissas deste estudo era a identificação de necessidades de formação na área das tecnologias digitais sentidas pelos diretores escolares, com particular incidência nas práticas profissionais dos mesmos. Deste modo, solicitou-se aos participantes a indicação das necessidades de formação, que posteriormente foram organizadas nas áreas/temáticas representadas no quadro 6 . 
Quadro 5 - Fatores potenciadores da integração educativa das tecnologias digitais na perspetiva dos diretores escolares

\begin{tabular}{lcc}
\hline \multicolumn{1}{c}{ FATORES PotenciadoREs } & n & $\%$ \\
\hline Professores inovadores & 91 & $68.42 \%$ \\
Valorização do trabalho dos professores & 73 & $54.89 \%$ \\
Mais software específico para as várias disciplinas & 70 & $52.63 \%$ \\
Mais ações de formação de âmbito disciplinar & 64 & $48.12 \%$ \\
Mais apoio técnico da escola, que pudesse auxiliar o professor nas suas & 60 & $45.11 \%$ \\
dificuldades & & \\
Ações de formação que prevejam a planificação de aulas utilizando & 55 & $41.35 \%$ \\
computador & 53 & $39.85 \%$ \\
Lideranças escolares inovadoras & 51 & $38.35 \%$ \\
Projetos educativos inovadores & 49 & $36.84 \%$ \\
Maior autonomia das escolas & 41 & $30.83 \%$ \\
Políticas educativas e incentivos estruturais & 33 & $24.81 \%$ \\
Flexibilidade dos currículos & 20 & $15.04 \%$ \\
Mais ações de formação de âmbito generalista & &
\end{tabular}

TOTAL $=133$

Quadro 6 - Necessidades de formação na área das tecnologias digitais apresentadas pelos diretores escolares

\begin{tabular}{llc}
\hline \multicolumn{1}{c}{ ÁREA/ TEMÁTICA } & $\mathbf{n}$ & $\%$ \\
\hline Plataformas de apoio à gestão e sistemas de avaliação & & \\
online & 26 & $15.79 \%$ \\
Folha de cálculo (Excel) & 22 & $16.54 \%$ \\
Bases de dados & 12 & $9.02 \%$ \\
Plataformas LMS (Eg. Moodle) & 11 & $8.27 \%$ \\
As TIC na gestão escolar & 7 & $5.26 \%$ \\
Ferramentas de trabalho colaborativo online & 6 & $4.51 \%$ \\
Criação de páginas web, blogues, sites & 6 & $4.51 \%$ \\
Redes informáticas e Intranets & 5 & $3.76 \%$ \\
Contabilidade/gestão & 4 & $3.01 \%$ \\
Tecnologias emergentes e inovadoras & 4 & $3.01 \%$ \\
Edição e tratamento de imagem & 4 & $3.01 \%$ \\
Apresentações eletrónicas & 4 & $3.01 \%$ \\
Quadros interativos & 3 & $2.26 \%$ \\
\hline Não sinaliza necessidades & 19 & $14.29 \%$ \\
\hline
\end{tabular}


Da análise do quadro 6 constata-se que os diretores escolares apresentam como principal necessidade a formação em áreas relacionadas com as práticas de gestão escolar, com particular relevância para a utilização de plataformas de apoio à gestão e sistemas de avaliação online (15.79\%), a utilização de folha de cálculo (16.54\%) e a criação e utilização de bases de dados $(9.02 \%)$. Em sentido oposto, surgem necessidades de formação relacionadas com práticas docentes ao nível da utilização de quadros interativos (2.26\%), apresentações eletrónicas (3.01\%) e edição e tratamento de imagem (3.01\%). Constata-se ainda que $14.29 \%$ não indicaram qualquer necessidade de formação.

\section{Conclusões}

Este estudo procurou analisar os níveis de proficiência e utilização das TD por parte dos diretores de escolas e agrupamentos de escolas do ensino público português nas práticas de gestão escolar, bem como identificar um conjunto de fatores condicionantes à plena integração das mesmas nos contextos escolares e sinalizar as necessidades de formação na área da temática em estudo apontadas pelos diretores escolares.

Os resultados encontrados permitiram concluir que os diretores escolares nacionais apresentam um grau de proficiência e um índice de utilização das TD favoráveis, nas várias dimensões da sua atividade profissional. Contudo, a utilização de formas de comunicação com recurso às novas tecnologias, como forma privilegiada de contacto entre os vários agentes educativos (pais, professores, alunos e órgão de gestão), apresenta um score médio relativamente reduzido e que, consequentemente, se entende por favorável estimular.

A análise da relação entre o grau de proficiência e o índice de utilização das TD revelou que as variáveis se correlacionam de forma expressiva e estatisticamente significativa. A associação entre as variáveis em causa revelou-se igualmente assinalável entre o grau de proficiência e as dimensões da escala de utilização das TD. Da análise dos dados, pode inferirse que a autoconfiança ou o grau de proficiência se apresenta associado à capacidade de utilização das tecnologias nas referidas dimensões, pelo que, para se promoverem mais e melhores regulações práticas da utilização das 
tecnologias, será igualmente necessário atender às crenças e conceções pessoais dos diretores escolares. Os resultados encontrados estão em sintonia com o trabalho de Abdullah, DeWitt, e Alias (2013) que referem também a importância das crenças pessoais dos diretores escolares enquanto fatores de influência na adoção e implementação das tecnologias digitais nas suas escolas. Nessa medida, referem os mesmos autores que diretores escolares com uma forte visão estratégica sobre o papel das tecnologias digitais na promoção de estratégias pedagógicas inovadoras assumem um papel preponderante na criação de uma cultura de utilização das tecnologias nos seus contextos escolares. Também Polly et al. (2010) consideraram que as práticas e as crenças dos diretores escolares são fatores importantes na definição e implementação de projetos pedagógicos inovadores suportados por tecnologias.

De igual modo, os dados tornam visíveis algumas necessidades de formação sentidas pelos diretores escolares, nomeadamente associadas ao seu envolvimento em ações de formação em plataformas e ferramentas de apoio à gestão, sistemas de avaliação online e aplicações de folha de cálculo. Segundo Afshari e Ghavifekr (2012), as competências dos diretores escolares no uso das tecnologias são um fator-chave para a criação de uma liderança tecnológica nos seus contextos escolares. Nesse sentido, os diretores devem compreender o papel das tecnologias nas suas práticas profissionais e envolver-se em iniciativas de formação que lhes permitam desenvolver competências necessárias para impulsionar o uso das tecnologias digitais pelos professores das suas escolas (Stuart et al., 2009).

Em relação aos fatores que, na opinião dos diretores escolares, maior impacto têm na integração das tecnologias nos seus contextos escolares, os dados destacam: a existência de professores inovadores; a valorização do trabalho dos professores; e a disponibilização de mais software e aplicações de cariz educativo. Desta forma, pode-se inferir que os diretores escolares consideram que são efetivamente as práticas docentes a exercer maior preponderância nos processos de integração das tecnologias nas suas escolas, ainda que ao mesmo tempo sinalizem outras necessidades existentes nas mesmas, nomeadamente a dificuldade em encontrar e mobilizar meios de valorização do trabalho dos professores e a falta de software e de aplicações específicas para as diferentes áreas disciplinares. 
Este estudo, ainda que de caráter descritivo e exploratório, apresentou resultados relevantes que necessitam de ser aprofundados e substanciados por mais amplas pesquisas nacionais e internacionais, através das quais se consiga perceber qual o papel dos diretores escolares nos processos de inovação e integração das tecnologias digitais nos seus contextos escolares. É importante salientar que "ultimately, as technology leaders, school administrators have (...) a large part of the responsibility in the implementation of the organization's shared vision and the effective integration of technology in the schools" (Cakir, 2012, p.279).

\section{Notas}

1 Não se conhecem dados relativos à implementação deste curso/ação de formação nem quantos diretores a frequentaram ou os efeitos conseguidos.

2 Em: http://tinyurl.com/lideresinovadores

\section{Referências}

Abdullah, N., DeWitt, D., \& Alias, N. (2013). School improvement efforts and challenges: A case study of a principal utilizing information communication technology. Procedia - Social and Behavioral Sciences, 103, 791-800.

Afshari, M., \& Ghavifekr, S. (2012). Transformational leadership role of principals in implementing informational and communication technologies in schools. Life Science Journal, 9(1), 281-284.

Afshari, M., Abu Bakar, K., Luan, W. S., Abu Samah, B., \& Fooi, F. S. (2008). School leadership and information communication technology. Turkish Online Journal of Educational Technology, 7(4), 82-91.

Anderson, R., \& Dexter, S. (2000). School technology leadership: Incidence and impact. (Teaching, Learning, and Computing: 1998 National Survey - Report \#6). Center for Research on Information Technology and Organizations, University of California, Irvine, and University of Minnesota.

Bandura, A. (1997). Self-efficacy: The exercise of control (1st ed.). New York: W. H. Freeman \& Co.

Bravo, C., \& Fernández, A. (2009). Integración curricular de los medios digitales en la formación docente. In J. P. Pons (Coord.), Tecnología Educativa. La formación del profesorado en la era de Internet (pp. 249-270). Málaga: Ediciones Aljibe.

Cakir, R. (2012). Technology integration and technology leadership in schools as learning organizations. The Turkish Online Journal of Educational Technology, 11(4), 273-282. 
Cassidy, S., \& Eachus, P. (2002). Developing the computer user self-efficacy (CUCE) scale: Investigating the relationship between computer self-efficacy, gender and experience with computers. Journal of Educational Computing Research, 26(2), 133-154.

Costa, F. A (Coord.). (2008). Competências TIC: Estudo de implementação (Vol. 1). Lisboa: Ministério da Educação - Gabinete de Estatística e Planeamento da Educação.

Creswell, J. W. (2003). Research design: Qualitative, quantitative and mixed methods approaches. London: SAGE Publications.

Creswell, J. W. (2010). Projeto de pesquisa. Métodos qualitativo, quantitativo e misto ( $3^{\mathrm{a}}$ ed.). Porto Alegre: Artmed.

Culp, K. M., Honey, M., \& Mandinach, E., (2003). A retrospective on twenty years of education technology policy. Disponível em http://www2.ed.gov/about/offices/ list/os/technology/plan/2004/site/docs_and_pdf/20yearsdocrevised.pdf (acesso em 12 de maio de 2014).

Daly, C., Pachler, N., \& Pelletier, C. (2009). Continuing professional development in ICT for teachers: A literature review. London: Institute of Education University of London.

DGAE (2013a). Códigos de escolas e agrupamento de escolas. Disponível em http://www.dgae.mec.pt/web/14654/171 (acesso em março de 2014).

DGAE (2013b). Programa líderes inovadores. Disponível em http://www.dgae.mec.pt/ web/14654/165 (acesso em maio de 2014).

DGRHE (2012). Programa líderes inovadores. Disponível em http://www.dgae.minedu.pt/web/14654/155 (acesso em abril de 2012).

Drent, M., \& Meelissen, M. (2008). Which factors obstruct or stimulate teacher educators to use ICT innovatively? Computers \& Education, 51, 187-199.

Fullan, M. R. (2001). The new meaning on educational change. New York: Teacher College Press.

Gooler, D., Kautzer, K., \& Knuth, R. (2000). Teacher competence in using technologies: The next big question. Honolulu: Pacific Resources for Education and Learning.

Hayes, D. (2007). ICT and learning: Lessons from Australian classrooms. Computers \& Education, 49, 385-395.

Hill, M. M., \& Hill, A. (2008). Investigação por questionário ( $2^{a}$ ed.). Lisboa: Edições Sílabo.

Ivers, K. S. (2002). Changing teacher's perceptions and the use of technology in the classroom. Comunicação apresentada no Annual meeting of the American Educational Research Association (AERA), New Orleans.

Jacobson, M., \& Hunter, W. (2004). Leadership and technology in schoolsDisponível em http://www.ucalgary.ca/ iejll/volume7/leadershipandtechnologyeditorial.htm (acesso em 20 de maio de 2014).

Liu, Y., \& Huang, C. (2005). Concerns of teachers about technology integration in the USA. European Journal of Teacher Education, 28(1), 35-47.

Miranda, G. (2007). Limites e possibilidades das TIC na educação. Sísifo, Revista de 
Ciências da Educação, 3, 41-50.

Mueller, J., Wood, E., Willoughby, T., Ross, C., \& Specht, J. (2008). Identifying discriminating variables between teachers who fully integrate computers and teachers with limited integration. Computers \& Education, 51(4), 1523-1537.

Mulkeen, A. (2000). The impact of teacher skills on the integration of ICT in Irish Schools. Disponivel em http://www.icte.org/To1_Library/T01_170.PDF (acesso em 14 de maio de 2014).

Norris, C., Soloway, E., \& Sullivan, T. (2002). Examining 25 years of technology in U.S. education. Communication of the ACM digital library, 45(8), 15-18.

OCDE (2001). Schooling for tomorrow learning to change: ICT in schools. Paris: OCDE.

Paraskeva, F., Bouta, H., \& Papagianni, A. (2008). Individual characteristics and computer self-efficacy in secondary education teachers to integrate technology in educational practice. Computers \& Education, 50(3),1084-1091.

Pedro, N. (2011). Utilização educativa das tecnologias, acesso, formação e autoeficácia dos professores (Tese de Doutoramento). Universidade de Lisboa, Lisboa.

Piedade, J. (2010). Utilização das TIC pelos professores de uma escola do ensino básico e secundário (Dissertação de Mestrado). Universidade de Lisboa, Lisboa.

Piedade, J., \& Pedro, N. (2012). (What produces the) best effects on teachers' ICT use in classroom: Formal training, informal training or neither? In Atas da International Conference on Information Communication Technologies in Education (pp. 103-113). Disponível em http:// http://www.icicte.org/ Proceedings2012/Papers/08-1-Piedade.pdf

Polly, D., Mims, C., Sheperd, C., E, \& Inan, F. (2010). Evicence of impact: Transforming teacher education with preparing tomorrow's teachers to teach with technology (PT3) grants. Teaching and Teacher Education, 26, 863-870.

Santos, A. P. (2010). Um estudo sobre a influência da formação nos índices de utilização efectiva das TIC e na auto-eficácia dos professores (Dissertação de Mestrado. Universidade de Lisboa, Lisboa.

Sweeney, B. (2005). Management and Leadership in Schools. Teacher: The National Education Magazine, Aug 2005, 48-50.

Stuart, L., H, Mills, A., M, \& Remus, U. (2009). School leaders, ICT competence and championing innovations. Computers \& Education, 52, 733-741.

Tearle, P. (2003). Enabling teachers to use information and communications technology for teaching and learning through professional development: Influential factors. Teacher Development, 7(3), 457-471.

Tondeur, J., Keer, H. V., Braak, J. V., \& Valcke, M. (2008). ICT integration in the classroom: Challenging the potential of a school policy. Computers \& Education, $51,212-223$.

Vannatta, R. A., \& Fordham, N. (2004). Teacher dispositions as predictors of classroom technology use. Journal of Research on Technology in Education, 36(3), 253271. 
Wong, E. M. L., \& Li, S. C. (2008). Framing ICT implementation in a context of educational change: A multilevel analysis. School Effectiveness and School Improvement, 19(1), 99-120.

Woodbridge, J. (2004). Technology integration as a transforming teaching strategy. Disponível em http://www.techlearning.com/shared/printableArticle.php? articleID=17701367 (acesso em 30 de janeiro de 2009).

Younie, S. (2006). Implementing government policy on ICT in education: Lessons learnt. Education and Information Technologies, 11, 385-400. 
DIGITAL TECHNOLOGIES IN SCHOOL ADMINISTRATION: PRACTICE, PROFICIENCY AND TRAINING NEEDS OF SCHOOL PRINCIPALS IN PORTUGAL

\section{Abstract}

This paper presents the results of a study organized according to the postpositivist research paradigm and is assumed a descriptive study involving 133 participants $(16 \%)$ of a universe of 831 principals of the Portuguese public schools. This study aims to analyze the practices and proficiency levels in digital technology (DT) use presented by the Portuguese school principals. Data collection process was based on the application of two online self-report scales: 'Computer self-efficacy scale' and the 'DT use in scholar administration practices scale', in the school year of 2012-2013. The results show favorable scores both in DT proficiency level and in the use of digital technologies in professional practices. Therefore schools principals reported favorable personal attitudes towards the use of DT in their own professional activities. The study also explores the principals' training needs in the area of digital technologies and even though the levels of DT use in professional practices are positive, principals pointed out significant needs regarding ICT training.

Keywords

ICT in education; School principals; Self-efficacy in DT use; DT use in school administration

\section{LAS TECNOLOGÍAS DIGITALES EN LA ADMINISTRACIÓN DE LA ESCUELA: LAS PRÁCTICAS, EL DOMINIO Y LAS NECESIDADES DE FORMACIÓN DE LOS DIRECTORES DE LAS ESCUELAS EN PORTUGAL}

Resumen

En este trabajo se hace la presentación de los resultados de un estudio de carácter descriptivo, desarrollado con 133 directores de escuelas de la educación pública de Portugal, donde se buscó analizar el índice de utilización 
de las tecnologías digitales (TD) en prácticas de administración de la escuela y el sentido de la autoeficacia en el uso de las mismas, presentados por los directores de escuela. El proceso de recolección de datos se estructura a partir de la aplicación de dos escalas: la 'Computer self-eficacy scale' y la 'Escala de uso de las TIC en las prácticas de administración de la escuela', en el año académico 2012-2013. Los resultados tienden a mostrar que los directores de escuelas tienen puntuaciones medias favorables de autoeficacia y el uso de TD en sus prácticas profesionales. El estudio también trató de explorar las necesidades de formación en el ámbito de la TD y, a pesar de las puntuaciones favorables de competencia y el uso de TD en las prácticas profesionales, los directores refieren necesidades de formación importantes.

\section{Palabras-clave}

Tecnologías digitales en la educación; Directores de escuela; Autoeficacia; Uso de TD en la gestión escolar

Recebido em junho/2014 Aceite para publicação em outubro/2014

i Instituto de Educação, Universidade de Lisboa, Portugal.

Toda a correspondência relativa a este artigo deve ser enviada para: Neuza Pedro, Instituto de Educação da Universidade de Lisboa, Alameda da Universidade, 1649-013 Lisboa. E-mail: nspedro@ie.ul.pt 